\title{
The Metallicity Distribution of Distant F and G Type Stars in the CFHT Legacy Survey Deep Field
}

\author{
Yüksel Karataş ${ }^{\mathrm{A}, \mathrm{C}}$, Mukremin Kilic ${ }^{\mathrm{B}}$, Orhan Güneş ${ }^{\mathrm{A}}$, and Füsun Limboz ${ }^{\mathrm{A}}$ \\ ${ }^{A}$ Istanbul University, Science Faculty, Department of Astronomy and Space Sciences, \\ 34119, Istanbul, Türkiye \\ B Department of Astronomy, The Ohio State University, Columbus, OH 43210, USA \\ ${ }^{\mathrm{C}}$ Corresponding author. Email: karatas@istanbul.edu.tr
}

Received 2008 June 23, accepted 2008 September 15

\begin{abstract}
We study the metal abundances of $F$ and $\mathrm{G}$ type stars in the Galactic disk and halo using the CanadaFrance-Hawaii Telescope Legacy Survey D4 field. For a sample of stars within $7 \mathrm{kpc}$ of the Galactic plane, we derive mean abundance values of $[\mathrm{Fe} / \mathrm{H}]=-0.77 \pm 0.36 \mathrm{dex}$ for the thick disk, and $[\mathrm{Fe} / \mathrm{H}]=-1.42 \pm 0.98 \mathrm{dex}$ for the stellar halo, respectively. These metallicites are consistent with the metallicity estimates from the Sloan Digital Sky Survey Data Release 6. We do not find a vertical abundance gradient for the thick disk between $1 \mathrm{kpc}$ and $4 \mathrm{kpc}$. However, stars within $1 \mathrm{kpc}$ of the Galactic plane are saturated in our dataset, and therefore we cannot rule out the observed vertical metallicity gradient for the thick disk stars in the SDSS. Moreover, we find a negative trend in the halo metallicity with increasing distance from the Galactic plane. This trend could be due to a contribution from an increasing number of very metal poor stars that belong to the outer halo. However, systematic effects in photometric metallicities are largest for the most metal poor stars, and therefore these effects cannot be ruled out.
\end{abstract}

Keywords: stars: abundances: stars: distances: techniques: photometric

\section{Introduction}

Our view of the formation of the Milky Way has changed dramatically since the discoveries of complex substructures in the Galaxy. The Milky Way has four spatially and kinematically distinct components; thin disk, thick disk, halo, and the bulge. However, recent discoveries of irregular structures like the Sagitarrius dwarf tidal stream and the Monoceros stream show that the formation of galaxies is not a steady process resulting in a smooth distribution of stars. Instead, galaxies are constantly shaped by the infalling smaller galaxies (see Juric et al. 2008 and the references therein).

The Sloan Digital Sky Survey (SDSS) and the Sloan Extension for Galactic Understanding and Exploration $(S E G U E)$ are providing photometry and spectroscopy for a very large number of stars that can be used to study the stellar populations in the Galaxy. Such a study involving $\approx 20000$ stars with the SDSS spectra demonstrated that the Galactic halo is likely to have two components; an inner and an outer component (Carollo et al. 2007). These two components seem to have different spatial density profiles, stellar orbits, and metallicities. Carollo et al. (2007) suggest that the outer halo formed through dissipationless chaotic merging of smaller subsystems within a pre-existing dark matter halo, supporting the complex galaxy formation scenarios. Ivezic et al. (2008) use the
SDSS photometry data to study the metallicity distribution of disk and halo stars. However, their dataset is not deep enough to test the dichotomy of the halo.

The Canada-France-Hawaii Telescope Legacy Survey (CFHTLS) provides a valuable new source to study the metallicity distribution of the Galactic stellar populations. The third data release of the Deep survey now provides ugriz photometry down to a limiting magnitude of 26 . CFHTLS lacks spectroscopic observations, which would provide the most accurate metallicities. However, imaging data can also be used to deduce stellar metallicities through the traditional UV excess method (Wallerstein 1962; Sandage 1969). The metal absorption lines mostly affect the UV part of the spectrum, therefore metal-rich and metal-poor stars with the same effective temperature (or the same $B-V$ color) can be differentiated based on their UV colors. In addition, imaging data enables us to obtain a complete flux-limited sample of stars and push the studies of stellar metallicities to fainter magnitudes than the spectroscopic surveys. Here we use the CFHTLS data for the D4 Field to perform such an analysis. Section 2 describes the data and the selection of point sources. The derivation of metallicites from the photometric data and its application to the CFHTLS ugriz system is discussed in Section 3, whereas the results from this analysis are discussed in Section 4. 


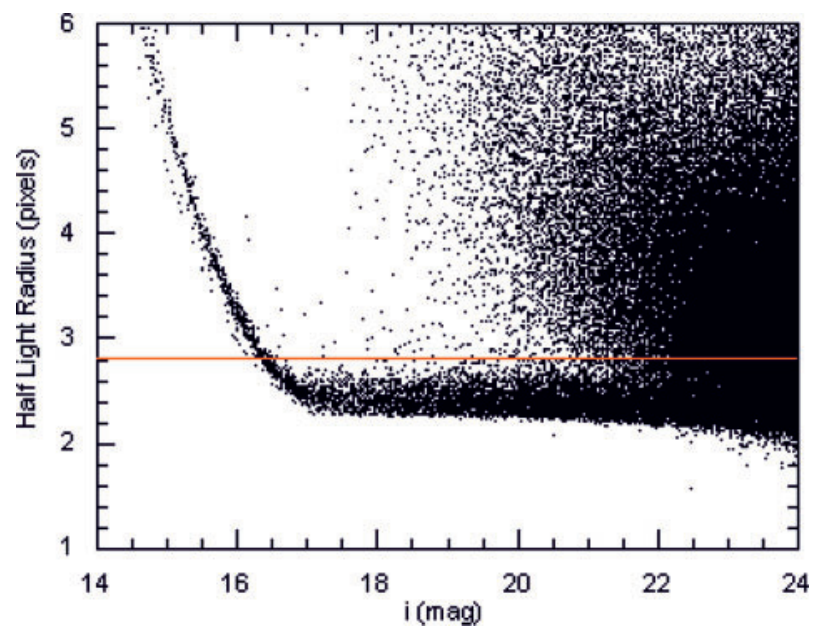

Figure 1 HLR versus $i$-band for the D4 field. Note that objects with $i<17.00$ are saturated. Star-galaxy separation seems to be robust down to $i=22 \mathrm{mag}$. The solid horizontal line marks the boundary for selection of point sources $(H L R<2.82)$.

\section{The Data}

The CFHTLS D4 field is located at RA $=22^{h} 15^{m} 31^{s}$, $\mathrm{DEC}=-17^{\circ} 43^{\prime} 56^{\prime \prime}(\mathrm{J} 2000), l=39^{\circ}$, and $b=-53^{\circ}$. The TERAPIX T003 public data release for the D4 field covers an area of $0.86 \mathrm{deg}^{2}$ and provides photometry with limiting AB magnitudes of $u=26.5, g=26.3, r=26.4, i=26.0$, and $z=25.0$, respectively. The high galactic latitude of this field is similar to the fields targeted by the SDSS.

Without follow-up spectroscopy, we have to rely on morphological classification to select point sources. Schultheis et al. (2006) and Limboz et al. (2008) showed that the star-galaxy separation is possible through the use of the half-light radius (hereafter, HLR) measurements from the $i$-band images. HLR corresponds to the radius that encloses $50 \%$ of the total flux from the source. Figure 1 shows the HLR versus $i$-band magnitudes for the sources in the D4 field. Objects with $i<17$ mag are saturated, and therefore have unreliable HLR measurements. Using spectroscopic data from the VLT-VIRMOS Deep Survey (VVDS; Le Fevre et al. 2005), Limboz et al. (2008) demonstrated that the spectroscopically confirmed stars have $\operatorname{HLR}(i)<2.82$ and that the star-galaxy separation is reliable down to $i=21 \mathrm{mag}$. Since the $C F H T L S$ goes five magnitudes deeper than this, we now look into pushing the point source classification limit to fainter magnitudes.

Figure 2 presents $(u-g)$ versus $(g-r)$ color-color diagrams for four different magnitude bins. The top left panel shows the color-color diagram for point sources (black points) and resolved sources (red points) for $i=$ 20-21 mag, where the point source classification seems reliable. This panel reveals a tight stellar sequence, and confirms the expectations that stars can be identified reliably down to $21 \mathrm{mag}$. The other panels in this figure show the color-color diagrams for $i=21-22,22-23$ and 23-24 mag. These three panels show that the stellar sequence is still clearly visible for $i=21-22$, but the galaxy contamination starts to become a problem for

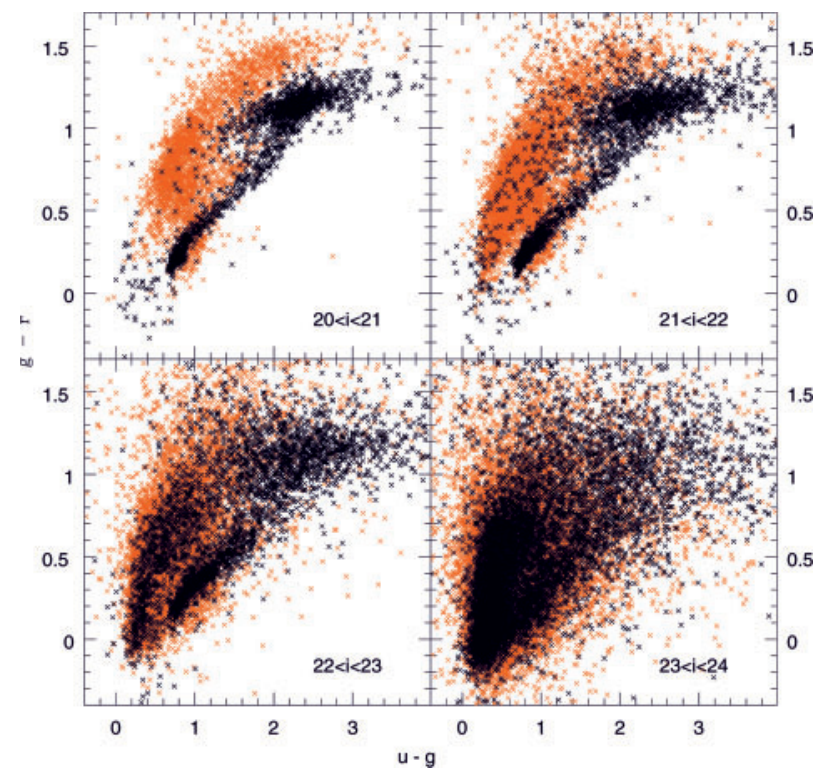

Figure $2(u-g)$ versus $(g-r)$ color-color diagram for different $i$-filter magnitude limits. Black points shows stars $(H L R<2.82)$, whereas red points represent galaxies $(H L R \geq 2.82)$.

$i>22$ mag. We count the number of sources that are classified as point-like and that lie to the left of the stellar sequence, and compare it with the number distribution of resolved sources with $\operatorname{HLR}(i)>2.82$ to estimate contamination of the stellar sequence by galaxies. We estimate that the galaxy contamination is less than $4 \%$ in the range $(g-r)=0.1-0.7 \mathrm{mag}$ for $i=21-22 \mathrm{mag}$. An analysis of the number distribution of resolved and un-resolved sources in the other panels show that the galaxy contamination ranges from $7 \%$ to $23 \%$ for $i=22-23 \mathrm{mag}$, and even higher for $i>23 \mathrm{mag}$. Therefore, we restrict our sample to the sources with $i<22 \mathrm{mag}$ and $H L R<2.82$. Using the above criteria, we classify 7348 sources as stars.

We use the dust maps of Schlegel et al. (1998) to deredden the photometry. $\mathrm{E}(B-V)$ for the $\mathrm{D} 4$ field ranges from 0.023 to $0.031 \mathrm{mag}$, with a mean of $0.027 \mathrm{mag}$. The small range of reddening correction for the D4 field shows that this correction does not have a signifincant effect on our analysis. Perhaps the most important correction required for the photometry is the correction from the MegaCam system to the standard ugriz filter system. Studying the differences between the ugriz system and the MegaCam system, Clem et al. (2008) find that the MegaCam griz filters are consistent with the standard system, though the $u$ band data need to be corrected using a third order polynomial. The MegaCam $u$-band filter was selected to take advantage of the better UV transparency of the $C F H T$ and it is slightly redder than the SDSS $u$-band filter. Clem et al. (2008) compare theoretical isochrones in the standard system and the corrected MegaCam system and they find that the isochrones overlap each other within 0.01 mag for both dwarfs and giants with colors $u-g=0.0$ to $+3.0 \mathrm{mag}$ and $g-i=-0.5$ to $+3.5 \mathrm{mag}$, and with metallicities $[\mathrm{Fe} / \mathrm{H}]=-2.3$ to 0.0 . We use the 

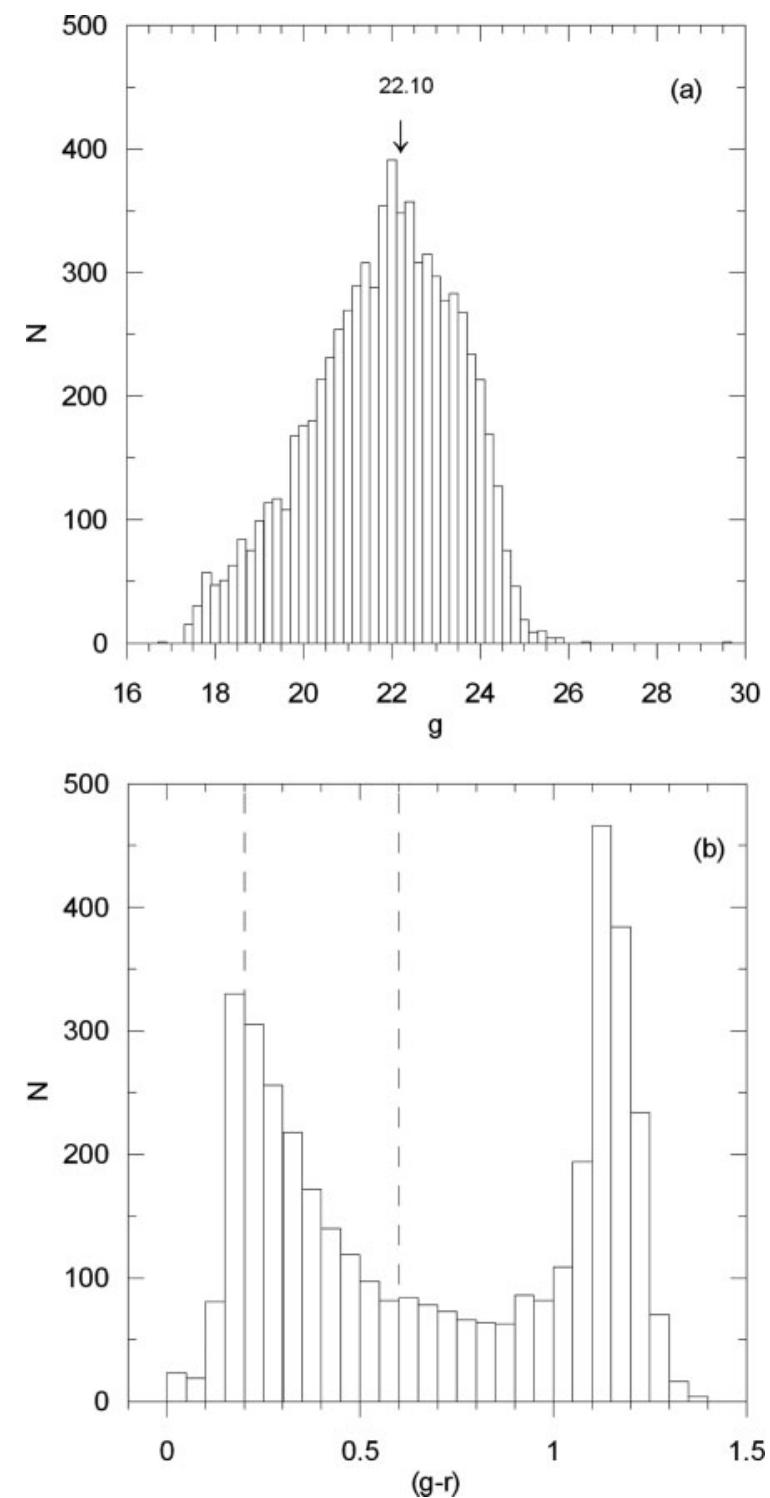

Figure 3 (a) $g$ magnitude distribution. Drop in $g=22.10$ is taken as the completeness limit. (b) $(g-r)$ distribution. The presence of the two main peaks at $(g-r) \sim 0.20$ and $\sim 1.15$ are seen. Vertical dashed lines show the color index range $0.20<(g-r)<0.60$, which corresponds to $\mathrm{F}$ and $\mathrm{G}$ type stars.

transformation given by Clem et al. (2008) (see their Figure 5) to convert the MegaCam $u$-band photometry into the standard $u$-band system. In addition, a correction is required to transform the photometry from the primed system to the SDSS $2.5 \mathrm{~m}$ telescope's unprimed u griz system. We use the transformations given by Tucker et al. (2006) to transform the photometry to the unprimed system.

The apparent $g$ magnitude distribution for our sample of stars is shown in Figure 3a. The number of stars increases with increasing magnitude until $g=22.1 \mathrm{mag}$, which we use as the completeness limit. For $\mathrm{a} \approx \mathrm{G} 5$ main-sequence star with $(g-r)=0.4$ mag (see Table 1 in Ivezic et al. 2008), this limit corresponds to a Galactocentric distance of $21 \mathrm{kpc}$ and a Galactic plane distance of $\approx 17 \mathrm{kpc}$. Figure $3 \mathrm{~b}$ shows the $(g-r)$ color distribution for our sample. There are two distinct peaks located at $\approx 0.20 \mathrm{mag}$ and $1.15 \mathrm{mag}$, corresponding to $\mathrm{F}$ stars and late type $\mathrm{M}$ stars, respectively. We select our sample of $\mathrm{F}$ and $\mathrm{G}$ type stars by restricting the sample to the stars with $0.20<(g-r)<0.60$ (shown by vertical dashed lines). This results in a sample of $1315 \mathrm{~F}$ and $\mathrm{G}$ stars. Majority of these stars lie above the synthetic $(u-g)$ versus $(g-r)$ relation of Pickles (1998), indicating that they are metal-poor disk and halo stars.

\section{The Metallicity Distribution}

Using SDSS spectroscopy and photometry, Ivezic et al. (2008) have developed a metallicity estimation method based on the $(u-g)$ and $(g-r)$ colors. Their method reproduces the metallicities obtained from low resolution $S D S S$ spectroscopy with a root-mean-square scatter of 0.2 dex. They also derive a photometric parallax relation using ugriz photometry of globular clusters. The agreement between the CFHT/MegaCam and the SDSS ugriz filter sets, after correcting the MegaCam u-band photometry (Clem et al. 2008), enables us to use their photometric metallicity and parallax methods on our dataset. Photometric metal abundances of the $\mathrm{F}$ and $\mathrm{G}$ dwarfs with $0.2<(g-r) \leq 0.4$, are determined via the following equation of Ivezic et al. (2008).

$$
\begin{aligned}
{[\mathrm{Fe} / H]=} & -4.37-8.56(u-g)+15.5(g-r) \\
& -39.0(u-g)(g-r)+23.5(u-g)^{2} \\
& +20.5(g-r)^{2}+12.1(u-g)^{2}(g-r) \\
& +7.33(u-g)(g-r)^{2}-10.1(u-g)^{3} \\
& -21.4(g-r)^{3}
\end{aligned}
$$

For $\mathrm{G}$ dwarfs with $(g-r)>0.4$, we replaced the $(u-g)$ color in the above equation with $(u-g)-$ $2(g-r)+0.80$. We estimate the absolute magnitudes of our sample of stars using the following relations from Ivezic et al. (2008).

$$
\begin{aligned}
M_{r}(g-i,[\mathrm{Fe} / \mathrm{H}])= & M_{r}(g-i)+\Delta M_{r}([\mathrm{Fe} / \mathrm{H}]) \\
\Delta M_{r}([\mathrm{Fe} / \mathrm{H}])= & 4.50-1.11[\mathrm{Fe} / \mathrm{H}] \\
& -0.18[\mathrm{Fe} / \mathrm{H}]^{2} \\
M_{r}(g-i)= & -5.06+14.32(g-i) \\
& -12.97(g-i)^{2}+6.127(g-i)^{3} \\
& -1.267(g-i)^{4}+0.0967(g-i)^{5}
\end{aligned}
$$

Figure 4 displays the $u-g$ color distribution and the derived metallicities for our sample of $F$ and $G$ stars. Both of these distributions are bimodal, and they are fairly well fitted with two gaussians. Ivezic et al. (2008) also found a bimodal $u-g$ color distribution for the SDSS sample with two peaks at $u-g \approx 0.9$ and $1.1 \mathrm{mag}$. The two peaks observed in the color distribution of our sample are located at $u-g=0.86 \pm 0.13$ and $1.10 \pm 0.41 \mathrm{mag}$. The agreement between the colors of our sample of $F$ and $G$ stars and that of the SDSS F and G stars shows that the 
Table 1. Metal abundances for the thick disk and halo stars as a function of distance from the Galactic plane

\begin{tabular}{lccccc}
\hline$z(\mathrm{kpc})$ & $\langle z\rangle$ & {$[\mathrm{Fe} / \mathrm{H}]_{\mathrm{THICK}}$} & $\sigma_{[\mathrm{Fe} / \mathrm{H}]}$ & {$[\mathrm{Fe} / \mathrm{H}]_{\mathrm{HALO}}$} & $\sigma_{[\mathrm{Fe} / \mathrm{H}]}$ \\
\hline$(1.0,2.0]$ & 1.5 & -0.73 & 0.30 & - & - \\
$(2.0,3.0]$ & 2.5 & -0.73 & 0.36 & -1.36 & 1.02 \\
$(3.0,4.0]$ & 3.5 & -0.71 & 0.29 & -1.36 & 0.88 \\
$(1.0,4.0]$ & 2.5 & -0.71 & 0.29 & -1.27 & 0.89 \\
$(4.0,8.0]$ & 6.0 & -0.85 & 0.38 & -1.57 & 0.96 \\
$(8.0,12.0]$ & 10.0 & -0.72 & 0.28 & -1.54 & 0.95 \\
$(12.0,16.0]$ & 14.0 & - & - & -1.77 & 0.69 \\
$(16.0,20.0]$ & 18.5 & - & - & -1.67 & 0.68 \\
$(20.0,27.0]$ & 23.5 & - & - & -1.65 & 0.62 \\
\hline
\end{tabular}
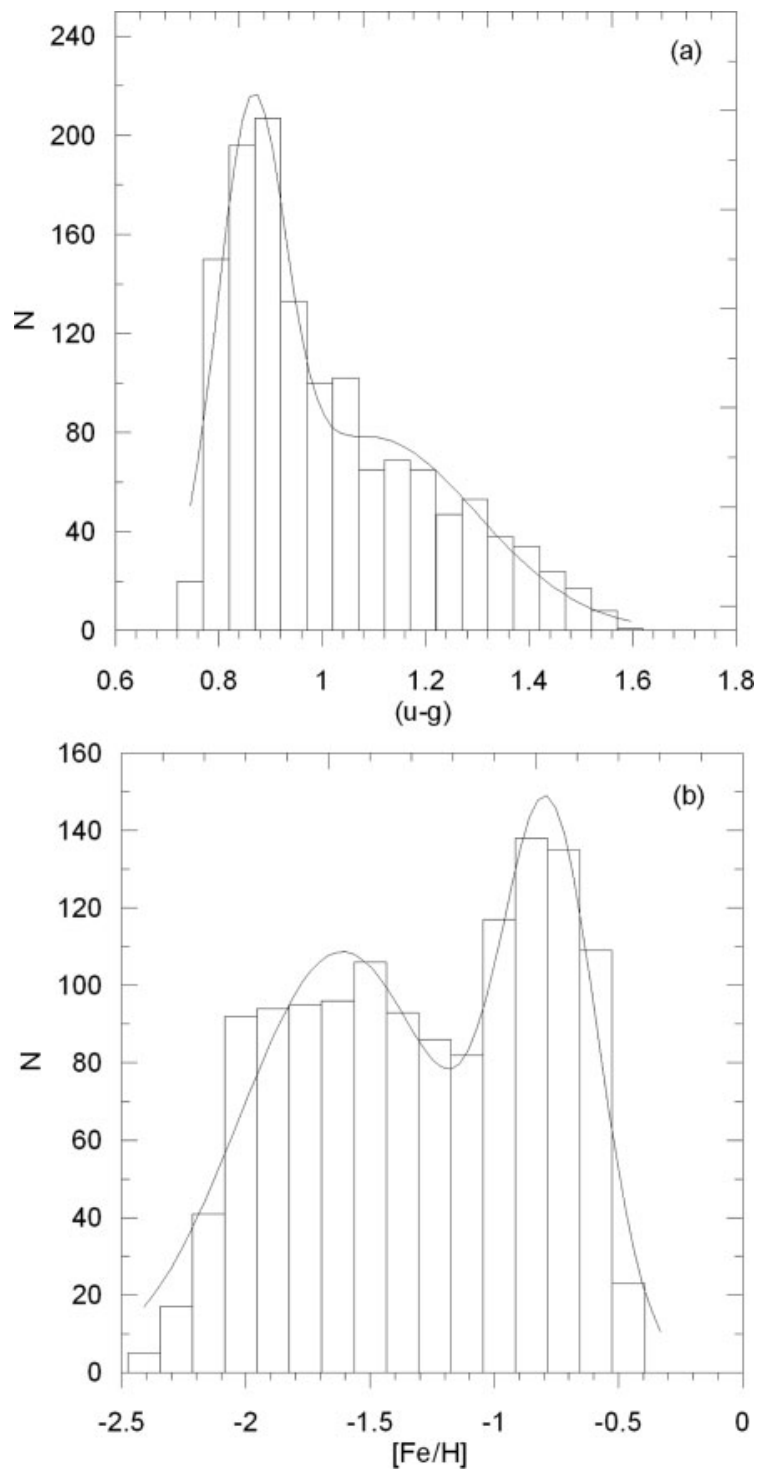

Figure 4 (a) $(u-g)$ color and (b) $[\mathrm{Fe} / \mathrm{H}]$ distribution for our sample of $1315 \mathrm{~F}$ and $\mathrm{G}$ type stars. Solid lines represent two Gaussian distributions fitted to the data.

transformations between the MegaCam photometry and the standard ugriz system are reliable. The two peaks in the $u-g$ color distribution correspond to metal-poor halo
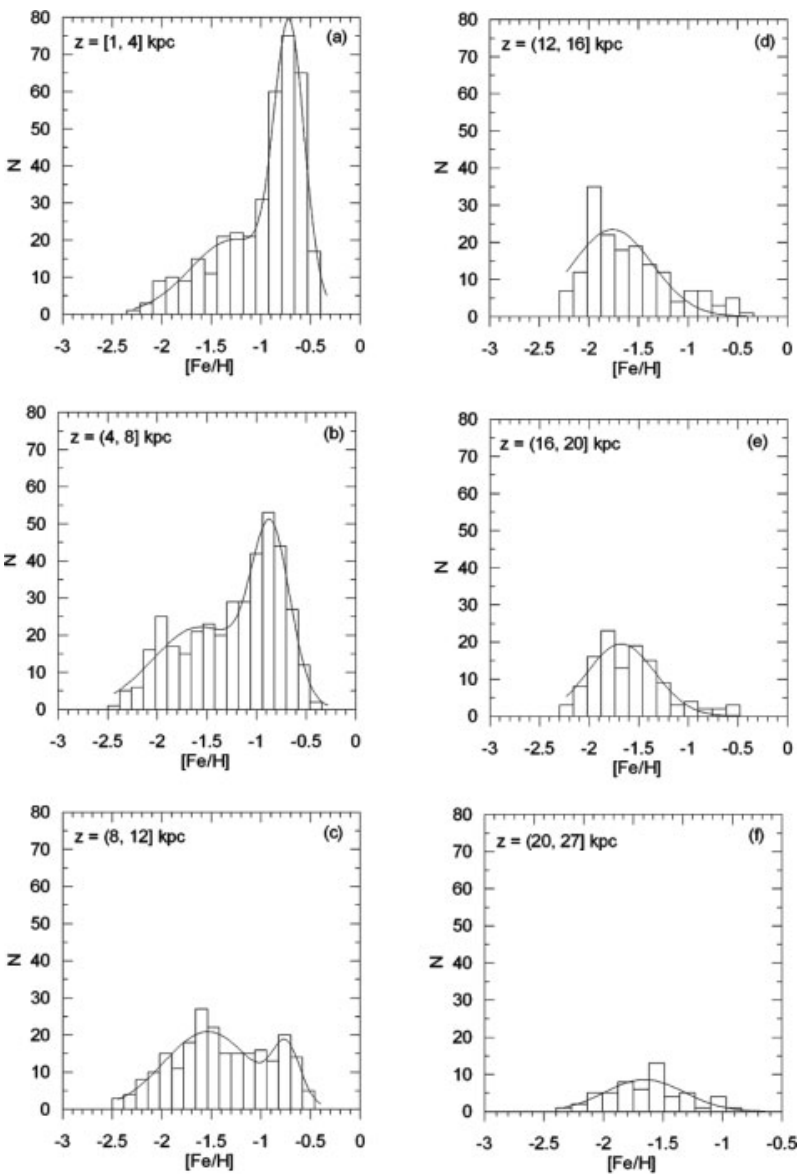

Figure 5 The metallicity distribution as a function of the distance from the plane of the Galaxy.

stars and more metal-rich and closer disk stars, respectively. The width of $0.13 \mathrm{mag}$ is significantly larger than the median $u-g$ error of 0.02 mag at $g=22 \mathrm{mag}$, providing a measure of the intrinsic variations in the $u-g$ color and the metallicity distribution.

The observed peaks in the $u-g$ color distribution translates into two peaks at $[\mathrm{Fe} / \mathrm{H}]=-0.78 \pm 0.39$ and $-1.62 \pm 0.82$ in the metallicity distribution. Figure 5 shows this distribution as a function of increasing distance from the Galactic plane. The mean metal abundances for the fitted gaussians are also listed in Table 1. The mean 
metal abundance shifts as a function of galactic plane distance. The thick disk dominates at $z=1-4 \mathrm{kpc}$, and a small contribution is still visible up to $8-12 \mathrm{kpc}$, after which only the halo stars with $[\mathrm{Fe} / \mathrm{H}] \approx-1.7$ are visible.

\section{Discussion}

Our mean metallicity estimate of $[\mathrm{Fe} / \mathrm{H}]=-0.78 \pm 0.39$ for the thick disk is consistent with the results from the analysis of $\mathrm{F}$ and $\mathrm{G}$ stars in the SDSS. Using the SDSS Data Release 3 spectroscopy, Allende Prieto et al. (2006) found that thick disk G dwarfs with $1<z<3 \mathrm{kpc}$ show a compact metallicity distribution with a peak at $[\mathrm{Fe} / \mathrm{H}] \approx-0.70$. Our mean metallicity estimates for $z=$ $1-2,2-3$, and 3-4 kpc (see Table 1) range from -0.71 to $-0.73 \mathrm{dex}$, and they are entirely consistent with the Allende Prieto et al. result. However, the mean metallicity for the thick disk is more metal-poor compared to the literature values of -0.60 (Gilmore \& Wyse 1985) and -0.50 (Carney et al. 1989). Since the SDSS photometric metallicity relation is known to underestimate the metallicity at high metallicities (see the discussion in Ivezic et al. 2008), our mean metallicity estimate for the thick disk probably suffers from these systematic effects.

Using the SDSS Data Release 6 photometry, Ivezic et al. (2008) found that the median metallicity for the thick disk smoothly decreases with distance from the plane from $[\mathrm{Fe} / \mathrm{H}]=-0.60$ at $500 \mathrm{pc}$ to -0.80 at several $\mathrm{kpc}$. We do not observe such a metallicity gradient for the thick disk between $z=1 \mathrm{kpc}$ and $4 \mathrm{kpc}$, but our mean metallicity estimate is consistent with their mean metallicity estimate beyond several $\mathrm{kpc},-0.80 \mathrm{dex}$. In fact, if we restrict our sample to $z<7 \mathrm{kpc}$, then the mean metallicity peak for the thick disk stars in our sample shifts to $[\mathrm{Fe} / \mathrm{H}]=-0.77 \pm 0.36$, entirely consistent with the Ivezic et al. analysis. Since stars with $i<17$ mag are saturated in the CFHTLS data, we do not have any stars within $1 \mathrm{kpc}$ of the Galactic plane. The trend found by Ivezic et al. is mostly based on the relatively brighter stars in their sample $^{1}$, which show a peak in metallicity at $[\mathrm{Fe} / \mathrm{H}] \approx-0.60$. The majority of the $z>1 \mathrm{kpc}$ thick disk stars in their sample show a peak around $[\mathrm{Fe} / \mathrm{H}] \approx-0.70$ (see their figure 7), similar to our analysis.

Figure 6 shows the metallicity trends for the thick disk and halo as a function of the Galactic plane distance. If we ignore the thick disk metallicity estimates beyond $8 \mathrm{kpc}$ due to small number statistics, then a small shift in metallicity is observed for the thick disk between 4 and $8 \mathrm{kpc}$. However, the mean metallicities for the stars with $z=1-4 \mathrm{kpc}$ and $4-8 \mathrm{kpc}$ are consistent within the errors. In addition, we do not find any significant trend between the Galactocentric distance and metallicity for the thick disk stars.

Our metallicity estimate of $-1.62 \pm 0.82$ for the halo is consistent with the literature values of $-1.60 \mathrm{dex}$ (Ryan \& Norris 1991) and -1.70 dex (Carney et al. 1996).

\footnotetext{
${ }^{1}$ This sample may be contaminated by metal-rich stars that belong to the high velocity tail of the thin disk distribution.
}

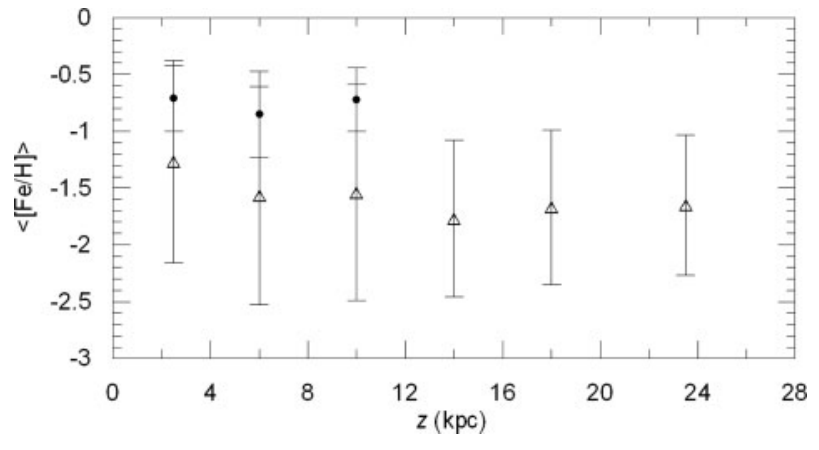

Figure 6 The mean metallicity for the thick disk (filled dots) and halo (open triangles) as a function of distance from the Galactic plane.

However, it is more metal poor than the halo metallicity derived using the SDSS F and G stars (Allende Prieto et al. 2006; Ivezic et al. 2008). Allende Prieto et al. (2006) found that the halo stars exhibit a broad range of iron abundances with a peak at $[\mathrm{Fe} / \mathrm{H}]=-1.40$, whereas Ivezic et al. (2008) find a peak at -1.46 dex with a standard deviation of 0.30 dex. Restricting our sample to $z<7 \mathrm{kpc}$, as in the Ivezic et al. study, we find that the mean metallicity for the halo stars within $z=7 \mathrm{kpc}$ is $[\mathrm{Fe} / \mathrm{H}]=-1.42$. Therefore, our metallicity estimates for both the thick disk and halo are consistent with the results from the SDSS analysis of stars within $z=7 \mathrm{kpc}$.

Since our results are consistent with the results from the SDSS, and the CFHTLS data goes deeper than the $S D S S$, we can use the CFHTLS data to study the stars more than $7 \mathrm{kpc}$ away from the Galactic plane. Our dataset is complete up to $z=17 \mathrm{kpc}$ for $g-r=0.4$ stars and up to $11 \mathrm{kpc}$ for the entire sample (see Section 2). Figure 6 shows that the mean metallicity for the halo decreases with increasing distance from the plane of the Galaxy. However, the metallicities are also consistent within the errors. If the decline in halo metallicity is real, this trend in metallicity and the observed broad distribution may be due to the inclusion of an increasing number of very metal poor stars in the halo.

Using spectroscopic and kinematic data from the $S D S S$ Data Release 5, Carollo et al. (2007) found a dichotomy in the metallicity distribution of the halo stars with two peaks at $[\mathrm{Fe} / \mathrm{H}] \approx-1.6$ and $[\mathrm{Fe} / \mathrm{H}]<-2.0$. They found that stars that reach large distances in their orbits above the Galactic plane show a metallicity peak around -2.1 dex, and they identified those stars as members of the outer halo. Since the number of these outer halo stars with retrograde orbits is small compared to the inner halo stars, it is impossible to identify them without kinematic information. However, a comparison of the observed metallicity distribution for our sample of stars and the fitted double gaussians in Figure 5 shows that the metal-poor tail end of the gaussian distribution for the halo underestimates the number of observed stars for $z>4 \mathrm{kpc}$. The majority of the panels in Figure 5 show a nearly flat distribution of stars between $[\mathrm{Fe} / \mathrm{H}]=-1.70$ and -2.0 . This could be caused 
by a contribution from the outer halo stars. However, without kinematic information, we cannot confirm or reject this idea. In addition, the systematic effects for the photometric metallicity estimates are largest for the most metal-poor stars. Ivezic et al. (2008) find that the root-mean-square scatter of the residuals between the photometric and spectroscopic metallicity estimates of the F and G stars in the $S D S S$ increases up to 0.30 dex for low metallicity stars. Even though we corrected for the differences between the MegaCam $u$ filter and the standard filter set, a small error in this transformation may also result in systematic offsets. For example, an offset of 0.04 mag in the $u$-band data can cause a metallicity bias of up to 0.20 dex (Ivezic et al. 2008). Hence, the observed distribution may be affected by these systematic problems.

\section{Conclusions}

We have performed a morphological selection of point sources and analyzed the metallicity distribution of distant $\mathrm{F}$ and $\mathrm{G}$ type stars in the CFHTLS D4 field. We have used MegaCam photometry transformed to the standard ugriz filter system, and used the photometric parallax and metallicity relations from the SDSS to obtain metallicities and distances for our sample of $1315 \mathrm{~F}$ and $\mathrm{G}$ type stars.

Limiting our study to $z=7 \mathrm{kpc}$, the distance limit used by Ivezic et al. (2008), we find that our estimates for the mean metallicity of the thick disk $([\mathrm{Fe} / \mathrm{H}]=-0.77)$ and halo $(-1.42 \mathrm{dex})$ are consistent with the results from the SDSS Data Release 6 photometry $(-0.80$ dex and -1.46 dex). Since the CFHTLS data is deeper than the $S D S S$, we are also able to check for trends in the thick disk and halo metallicity distribution beyond $7 \mathrm{kpc}$. Unlike the Ivezic et al. study, we do not find a trend in metallicity for the thick disk between 1 and $4 \mathrm{kpc}$. However, the significance of the trend observed in the SDSS dataset comes from the stars within $1 \mathrm{kpc}$ of the Galactic plane. Unfortunately, those stars are saturated in the CFHTLS observations, and the metallicity trend for the thick disk stars further away than $1 \mathrm{kpc}$ from the plane is consistent with the $S D S S$ results.

We observe a decline in metallicity of the halo with increasing distance from the Galactic plane. We suggest that this decline may be due to an increasing contribution from the very metal poor $([\mathrm{Fe} / \mathrm{H}]<-2)$ halo stars, but we cannot rule out systematic problems in our analysis. Proper motion data from the CFHTLS observations will be available at the end of the survey period. These data will be useful to study the kinematic properties of our sample of stars and constrain the contribution of very metal poor stars.

\section{Acknowledgments}

We thank D. An for useful discussions, C. Benoist for providing us with the CFHTLS data, and the anonymous referee for useful comments. This work is partly based on observations obtained with MegaPrime/MegaCam, a joint project of CFHT and CEA/DAPNIA, at the CanadaFrance-Hawaii Telescope (CFHT) which is operated by the National Research Council (NRC) of Canada, the Institut National des Science de l'Univers of the Centre National de la Recherche Scientifique (CNRS) of France, and the University of Hawaii. This work is based in part on data products produced at TERAPIX and the Canadian Astronomy Data Centre as part of the Canada-FranceHawaii Telescope Legacy Survey, a collaborative project of NRC and CNRS.

\section{References}

Allende Prieto, C., Beers, T. C., Wilhelm, R., Newberg, H. J., Rockosi, C. M., Yanny, B. \& Lee, Y. S., 2006, ApJ, 636, 804

Carney, B., Latham, D. W. \& Laird, J. B., 1989, ApJ, 97, 423

Carney, B., Laird, J. B., Latham, D. W. \& Aguilar, L. A., 1996, AJ, 112,668

Carollo, D. et al., 2007, Nature, 450, 1020

Clem, J., VandenBerg, D. A. \& Stetson, P., 2008, AJ, 135, 682

Gilmore, G. \& Wyse, R. F. G., 1985, AJ, 90, 2015

Helmi, A. et al., 2003, ApJ, 586, 195

Ivezic, Z. et al., 2008, ApJ, 684, 287

Le Fevre, O. et al., 2005, A\&A, 439, 845

Limboz, F., Karatas, Y., Kilic, M., Benoist, C. \& Alis, S., 2008, MNRAS, 383, 957

Ryan, S. G. \& Norris, J.E., 1991, AJ, 101, 1865

Schlegel, D. J., Finkbeiner, D. P. \& Davis, M., 1998, ApJ, 500, 525

Schultheis, M., Robin, A., Reyle, C., McCracken, H. J., Bertin, E., Mellier, Y. \& Le Fevre, O., 2006, A\&A, 447, 185

Pickles, A. J., 1998, PASP, 110, 863

Tucker, D. L. et al., 2006, AN, 327, 821

Sandage, A., 1969, ApJ, 158, 1115

Wallerstein, G., 1962, ApJS, 6, 407 